\title{
Gas balance in ASDEX Upgrade with Tungsten first wall
}

\author{
V. Rohde*a , V. Mertens ${ }^{a}$, A. Scarabosio ${ }^{\text {a }}$, \\ ASDEX Upgrade Team, \\ ${ }^{a}$ Max-Planck-Institut für Plasmaphysik, EURATOM Association, Boltzmannstr. 2, \\ D-85748 Garching, Germany
}

\begin{abstract}
Gas balance investigations were performed at the strongly pumped divertor tokamak ASDEX Upgrade. The gas inlet and pumping systems have been recalibrated, reaching an accuracy of about $3 \%$, which is mostly given by uncertainties of the gas temperature. Clear saturation of the wall is observed after the wall is loaded with $19 \cdot 10^{21}$ at almost independent from details of the discharge program. For a saturated wall the retention of $1.5 \pm 3.2 \%$ is found. In former carbon wall experiments wall saturation is also found, but a higher D-retention is observed.
\end{abstract}

Preprint submitted to Elsevier Science

28 August 2008 
PACS:52.40 Hf, 52.25 Xz, 52.55.Fa, 52.55.Rk

JNM keywords:First Wall Materials, Plasma Material Interaction, Tungsten, Hydrogen

PSI-18 Keywords: ASDEX Upgrade, Deuterium inventory,Gas injection \& Fueling, Tungsten, Wall pumping

* Corresponding author address: Max-Planck Institut für Plasmaphysik, Boltzmannstr. 2, 85478 Garching, Germany

* Corresponding author e-mail: Volker.Rohde@ipp.mpg.de presenting author: Volker Rohde presenting author e-mail:Volker.Rohde@ipp.mpg.de 


\section{Introduction}

One criterion for the choice of the plasma facing components (PFCs) in ITER is the expected retained tritium, which may exceed the maximal allowed inventory. Present investigations in limiter devices with carbon PFC found retention up to $50 \%$ of the injected gas even for long duration pulses [1]. High retention up to $40 \%$ was also observed for low density, high power discharges at divertor tokamaks [2]. At ASDEX Upgrade (AUG), a strongly pumped divertor tokamak, gas puff rates of typical $5 \cdot 10^{22} a t / s$ are applied to reach detached divertor conditions. Most of the puffed gas is transported via the open field lines into the divertor, where it is neutralized and pumped. For this purpose AUG is equipped with 14 turbo molecular pumps (TMP) and an in-vessel cryo pump $(\mathrm{CP})$. During the last years the PFCs in AUG have been changed from carbon to tungsten coated carbon [3],[4]. All PFCs had been cleaned to remove layers (consisting out of $\mathrm{B}, \mathrm{D}$ and $\mathrm{O}$, less than $10 \% \mathrm{C}$ ) from preceding campaigns during the last vent. No wall coating as boronisation, which would act as an additional getter, had been applied for wall conditioning during the discharges presented in this paper. For comparison some older discharges with carbon dominated wall and boron layers are shown.

Two alternative approaches are used to measure the deuterium retained in the vessel. The most comprehensive data base exists for probe analysis [5]: tiles are removed and analyzed using surface sensitive techniques. Typical $4 \%$ of the injected gas had been found, mostly in redeposited a:C-D layers, for carbon PFCs, only $0.3 \%$ for full tungsten PFCs [6]. This technique offers, due to the limited number of probes, a lower limit of the retained hydrogen inventory. Alternative approaches are gas balances, presumably the only diagnostic on 
retained gas during the $\mathrm{H}$ and $\mathrm{D}$ phase of ITER. Whereas probe analysis give a weighted average of the whole campaign, gas balances investigations offer temporal resolution over a single discharge as well as on a shot to shot base, this is mandatory to investigate different plasma scenarios.

\section{Experimental setup}

The basic idea of gas balances is to determine the difference of gas input and removed gas. In AUG most of the injected gas is pumped during the discharge, which requires high accuracy measurements for gas balances. As basis for these measurements we use calibrated capacitive gauges. The volume of the vessel was determined by expanding the inventory of a known volume into the vessel for closed valves, which leads to a static volume of $41562 \pm 100 l$. The second step had been a calibration of the gas inlet valves: A preprogrammed amount of gas was puffed into the vessel and compared to the amount determined by the pressure rise. The calibration done in 2003 has been repeated in 2008, leading to a correction of typical $2 \%$. The pumping speed of the CP had been determined dynamically with respect to the divertor gauge. Additionally, a known amount of gas was puffed and pumped. Then the LHe shield was regenerated into the vessel to get an accurate static measurement. The gauge is not mounted at the position of the cryo pump, so the same procedure was done for plasma discharges. As the effective pumping speed of the CP depends on the pressure, the calibration was performed for different plasma scenarios and a linear function was fitted to the total amount of gas found during the LHe warm up. The pumping speeds of the TMP and Neutral Beam Injection [NBI] boxes were determined by the dynamic pressure reached using a calibrated 
gas puff. The influence of pumps mounted at diagnostics turned out to be negligible. All used pumping speeds are summarized in Tab 1.

From gas kinetics it is known that the amount of gas $\mathrm{N}$ is determined by

$$
p V=N R T
$$

with $\mathrm{p}$ the pressure, $\mathrm{V}$ the volume, $\mathrm{R}$ the gas constant and $\mathrm{T}$ the temperature. Up to now we assume a constant temperature of the gas, which may be not valid after a plasma discharge. The $L N_{2}$ shielding of the cryo pump is kept always at low temperature, whereas parts of the main chamber, like limiters, are heated up during the discharge. To get the effective gas temperature a known amount of gas was puffed into the vessel. The resulting pressure rise allows estimating the gas temperature. Comparing the data for warm and cold $L N_{2}$ shield of the cryo pump a temperature difference of $-7 \mathrm{~K}$ was observed for the divertor measurements, leading to a correction of $2 \%$ for the amount of gas. After a plasma discharge the situation is more complex: Parts of the vessel, for example the protection limiters, are heated. Again a gas puff $5 \mathrm{~min}$ after a discharge was used to get the effective temperature. Depending on the shot scenario temperature differences between $-5 K$ and $+10 K$ are found. Although the database of discharges with these measurements is limited up to now, we found that the error in the gas temperature restricts the gas balance after a discharge to about $2 \%$. 


\section{$3 \quad$ High gas flux discharges}

Semidetached H-mode discharges are foreseen to be the most relevant for ITER, we concentrate on high density discharge. In AUG a standard H-mode discharge, which is performed on each experimental day, can be used as a basis for gas balances. This $1 \mathrm{MA}$ shot is heated with $5 \mathrm{MW}$ of NBI, reaches line averaged densities of $9 \cdot 10^{19} \mathrm{~m}^{-3}$ and total gas puff of $1 \cdot 10^{23} \mathrm{at}$ during 6 seconds. As gas input we used the sum of the flux from all gas valves $\Phi_{\text {valves }}$ and the particle flux of the NBI boxes $\Phi_{\text {Beam }}$. The gas is removed by the CP $\Phi_{C P}, \mathrm{TMP} \Phi_{T M P}$ and NBI boxes $\Phi_{N B I}$. Additionally the actual inventory of the plasma discharge $N_{p l}$ and the neutral gas in the vessel $N_{n t}$ is considered. The ratio of these quantities leads to the gas balance $R_{D}$ :

$$
R_{D}=\left(\int\left(\Phi_{C P}+\Phi_{T M P}+\Phi_{N B I}\right) d t+N_{p l}+N_{n t}\right) / \int\left(\Phi_{\text {valves }}+\Phi_{\text {Beam }}\right) d t(2)
$$

These quantities are plotted for the typical shot\# 22974 in Fig. 1a. The discharge $\left({ }^{s h o t}\right)$, the time when the plasma current is greater than $200 \mathrm{kA}$, lasts till $6.5 \mathrm{~s}$. At $8 \mathrm{~s}$ a gas pulse is applied for diagnostic calibration purpose. The plasma discharge consists out of the limiter break down ( ${ }^{\lim }$,from $0.0 \mathrm{~s}$ to $0.3 \mathrm{~s})$, plasma ramp-up ( ${ }^{r a m p}$,from $0.3 \mathrm{~s}$ to $\left.1.2 \mathrm{~s}\right)$, high gas puff phase $\left({ }^{h i g h}\right.$, from $1.2 \mathrm{~s}$ to $5.5 \mathrm{~s}$ ) and a smooth ramp down (till $6.5 \mathrm{~s}$ ). The retention factors for the different phases are summarized in table 2. During the limiter phase gas is released from the central column, but as the amount is low it has no influence on the total balance. Fig. 1 b shows the amount of the puffed gas, pumped gas and plasma inventory. During the ramp up $87 \%$ of the gas is retained, whereas $9 \%$ are used for plasma density build up, resulting in a loading of the PFCs [7]. After this phase the plasma density is almost constant, but the amount 
of pumped gas is still rising, until it reaches constant values ( ${ }^{\text {load }}$, from $0.0 \mathrm{~s}$ to $2.9 \mathrm{~s})$. Then steady state conditions $\left({ }^{p=p}, 2.9-4.0 \mathrm{~s}\right)$ are reached: within the error bars the same amount of gas is pumped as puffed. The integrated inventory is illustrated in Fig. 1 c. Up to $2.9 \mathrm{~s}$ gas is retained until $18 \cdot 10^{21}$ at are stored at the PFCs. A small rise of the pumped gas during the flat top phase is presumably due to outgasing of heated PFCs. After the discharge most of this gas is released within $30 \mathrm{~s}$ ( ${ }^{\text {short }}$, from 0 to $30 \mathrm{~s}$ ).

To get some better statistics, 30 shots with the highest gas inventory of the 2008 campaign before the boronisation have been investigated. Discharges with a major disruption or after overnight HeGD had been not taken into account. From earlier investigations it is known that uncoated W PFCs stores significant amounts of He [8]. As the cryo pump does not pump He, the determined pumping speed would be wrong in this case. The total gas input during the shot and amount of pumped gas in shown in Fig. 2. Taking the average of all these discharges we found a retention during the shot ( $\left.{ }^{s h o t}: I_{p}>200 k A\right)$ of $1-R_{D}^{\text {shot }}=8.2 \pm 3.3 \%$ and a short term retention of $1-R_{D}^{\text {short }}=3.6 \pm 2.7 \%$.

During the high density phase only $1-R_{D}^{P=P}=1.5 \pm 3.2 \%$ of the puffed gas is retained. To reach this phase a gas puff of $\int \Phi^{\text {load }}=54 \pm 6 \cdot 10^{21}$ at is needed, leading to a wall inventory of $N_{\text {wall }}=19 \pm 1 \cdot 10^{21}$ at. Taking the retention during the shot $N_{\text {wall }}^{\text {shot }}=1.2 \cdot 10^{21} \mathrm{at} / \mathrm{s}$ is found.

\section{Global gas balances}

To clarify the behavior of the gas balance the procedure mentioned above has been applied to all discharges of the 2008 campaign. $R_{D}$ was calculated automatically for the time of the shot, as defined above. Discharges with a 
length of less than $1 \mathrm{~s}$ and after the boronisation are not taken into account. In total 338 useful shots were investigated. In a second step 19 shots, which were identified to have a high He content (see above), without cryo pump, with significant fueling by pellets (uncertainties of amount) and high performance improved H-mod discharges (wall heating) were deselected. $R_{D}$ for all other discharges is shown in Fig. 3. The experimental campaign started after pump down and baking with shot \#22650. During the vent the remnants of former boronisations had been removed from PFCs. Only HeGD was applied for initial conditioning, no wall coating. For the first 100 discharges strong outgasing of the wall is observed. As the maximum baking temperature in AUG is restricted to $150^{\circ} \mathrm{C}$ the PFCs has to be conditioned by plasma discharges. For this purpose, a standard shot was repeated with increasing heating power. After this initial conditioning $R_{D}$ shows a scatter of values always below 1 . Taking the average of these discharges $90 \%$ of the puffed gas is pumped during the shot. In total $\sum R^{\text {shot }}=1.1 \cdot 10^{24}$ at were retained during this $1750 \mathrm{~s}$ of plasma discharges. As mentioned above the majority of this amount is outgased inbetween two discharges.

More interesting is the fluence dependence of $R_{D}$. The same set of data starting after the initial conditioning $(\# \geq 22700)$ is plotted versus the total gas puff in Fig. 4. For low fluxes a linear growth of the ratio with a strong scatter is observed. Higher fluxes show a clear saturation. From the evaluation of a single shot, as mentioned above, a saturation is expected for a flux of $5.4 \pm 0.6 \cdot 10^{22} a t$. This agrees well with the global data set. It is worth mentioning that all discharges had been taken into account, i.e. the wall saturation is independent on different plasma scenarios. 


\section{Carbon versus tungsten}

Gas balances had been presented for carbon dominated PFCs in 2003 [9] and tungsten main chamber PFCs in 2007 [10]. For comparison a shot with similar plasma parameters as used above has been re-evaluated using the new calibration. Unfortunately the divertor in AUG was changed after 2003. As this affects mostly the conductance from the outer strike point to the cryo pump, and the pressure at the cyro chamber is used to calculate the amount of pumped gas, the new calibration could be applied to the former data, keeping in mind, that the error bars will be bigger. To compare the different calibrations the same time intervals $\left({ }^{12 s}\right)$ as in [9] was used for data evaluation. The new calibration yields for $\# 17351 R_{D}^{12 s}=0.78$, whereas the former one yielded $R_{D}^{12 s}=0.72$. The results for similar discharges during the carbon phase (\#17351), the tungsten main chamber, but carbon radiation dominated phase ( \#20447) and full tungsten phase are compiled in table 3. During the limiter phase much less gas is released from the tungsten PFCs. Comparing only the high density phase shows that for the carbon PFCs about $19 \%$ of the gas is retained, whereas for clean tungsten PFC almost no additional inventory is built up. This results in different values of $R_{D}^{\text {shot }}$ for the plasma discharge. Retention for carbon PFCs seems to be higher than for tungsten. Wall saturation depends on the amount of gas puffed, which was less for carbon PFCs. Adding the data of \#17351 and \#20447 to the global plot of the last campaign (Fig.4) they match the new data, but are located at the lower values. Unfortunally a direct comparison of the C and W PFC's is not possible, as for the carbon data shots using the highest puffing rates are missing and no shots without boron layers had been performed. 


\section{Discussion}

The gas inlet and pumping systems of AUG has been recalibrated to reach maximum accuracy. Although the pressure gauges provide very accurate data, the unknown temperature of the pumped gas turned out to limit gas balance measurements in AUG to accuracy about 3\%. As parts of the vessel are cooled down to $L N_{2}$ temperature and parts as protection limiters of diagnostics are heated up by plasma discharges the uncertainty in the gas temperature turned out to be the main error. Since probe measurements indicate $1-R_{D} \leq 1 \%$ the current accuracy of the gas balance is not yet sufficient to yield comparable conclusions. To enhance the accuracy an additional storage volume is needed, with stores all pumped gas with at a defined temperature. Currently it is under discussion to install such a device at AUG. To complete this investigations the long term outgasing and the residual gas composition have been investigated.

The gas balance investigations with clean tungsten PFCs yield two important findings. First the retained gas 30 seconds after discharges is less than a few percent of the injected gas. Secondly clear wall saturation is observed. The main goal of gas balance investigations is to estimate the expected $\mathrm{T}$ retention in ITER. ITER has to operate with detached divertor conditions to avoid excessive erosion at the strike points. The predicted divertor neutral pressure is higher than in AUG. Even as ITER will be less pumped as AUG, we assume that a strong gas puff is needed and, similar to AUG, most of the gas will be pumped. The shot length in AUG is too short to reach steady state conditions concerning the wall temperature as shown in Tore Supra. On the other hand the gas puffing rate is two orders of magnitude higher. In AUG clear wall saturation is observed with a maximum retention of $1.9 \pm 0.1 \cdot 10^{22}$ at. After 
this, within the error bar of a few percent, all gas puffed is pumped away, i.e. the amount of retained gas does not depend on the length of the discharge. Wall saturation was also found for carbon PFCs, but the retention is always significant. Unfortunally no data using the highest puffing rate are avaiable for carbon PFC. Whereas for full tungsten, most of the gas is outgassed on a $30 \mathrm{~s}$ time scale the outgasing lasts much longer for carbon wall.

The gas balance shows much clearer behavior, after the wall was cleaned from remnants of former boronisations. In AUG the carbon wall was always operated with an active boronisation. The boronisation acts as in vessel getter pump strongly depending on history. Unfortunally, data for high flux divertor devices with an uncoated carbon wall are not available.

\section{Acknowledgement}

The authors would like to thank the European Task Force on Plasma Wall Interaction for stimulating these investigations.

\section{References}

[1] T. Loarer these Proceedings, R-3.

[2] P. Coad et al., Physica Scripta, T81, (1999), 7

[3] R. Neu et al.,PPCF 49, (2007), B59

[4] R. Dux et al.,these Proceedings, I-6.

[5] M. Mayer et al., Nucl. Fusion, 47 (2007), 1607

[6] M. Mayer et al.,these Proceedings, I-13. 
[7] G. Haas et al.,J. Nucl. Mat., 121 (1984), 151

[8] K. Schmid, Nucl. Fusion, 47 (2007), 984

[9] V. Mertens et al., ECA 27A, P-1,128, (2003)

[10] V. Rohde et al., ECA 31F, P-2.030, (2007) 
Table 1:

\begin{tabular}{|c|c|}
\hline Pump & $D_{2}$ pumping speed $[\mathrm{l} / \mathrm{s}]$ \\
\hline & $1 \cdot 10^{-5}$ mbar $\leq \mathrm{p} \leq 1 \cdot 10^{-2}$ mbar \\
\hline Turbomolecular Pumps & $6.85 \cdot 10^{3}+5.0 \cdot 10^{3} \cdot p$ \\
\hline Cryo pump & $1.15 \cdot 10^{5}+1.5 \cdot 10^{7} \cdot p$ \\
\hline NBI Box 1 & $2.9 \cdot 10^{4}+5 \cdot 10^{6} \cdot p$ \\
\hline NBI Box 2 & $2.3 \cdot 10^{4}+4.1 \cdot 10^{6} \cdot p$ \\
\hline Diagnostics & $\leq 500$ \\
\hline
\end{tabular}

Table 1: Used effective pumping speeds as a function of the pressure $\mathrm{p}$ [mbar].

Table 2:

\begin{tabular}{|c|c|c|c|c|c|c|}
\hline Phase & $T_{\text {start }}$ & $T_{\text {end }}$ & $N_{\text {input }}$ & $N_{\text {out }}$ & $\Delta N_{\text {plasma }}$ & $R_{D}$ \\
\hline & $\mathrm{s}$ & $\mathrm{S}$ & $10^{20} a t$ & $10^{20} a t$ & $10^{20} a t$ & \\
\hline limiter & 0.0 & 0.3 & 2.2 & 0.7 & 2.2 & 1.35 \\
\hline ramp-up & 0.3 & 1.2 & 85 & 2.2 & 8.0 & 0.12 \\
\hline $\mathrm{p}=\mathrm{p}$ & 2.9 & 4.0 & 383 & 384 & -0.3 & 1.0 \\
\hline shot & 0.35 & 6.2 & 915 & 869 & - & 0.94 \\
\hline short & -1.0 & 30 & 947 & 932 & - & 0.98 \\
\hline
\end{tabular}

Table 2: Gas balance of the different phases of \# 22974. 
Table 3:

\begin{tabular}{|c|c|c|c|c|c|c|c|}
\hline shot & wall & $R_{D}^{\text {lim }}$ & $N_{\text {Plasma }}$ & $\Phi_{\text {puff }}^{\text {pup }}$ & $R_{D}^{\text {high }}$ & $\Phi_{\text {puff }}^{\text {shot }}$ & $R_{D}^{\text {shot }}$ \\
\hline & & & $\cdot 10^{19} m^{-3}$ & $\cdot 10^{20} a t$ & & $\cdot 10^{20} a t$ & \\
\hline 17351 & C,B & 4.38 & 11.8 & 207 & 0.81 & 551 & 0.74 \\
\hline 20447 & W,C,B & 1.30 & 7.1 & 307 & 0.96 & 1090 & 0.88 \\
\hline 22974 & W & 1.36 & 10.3 & 280 & 1.0 & 915 & 0.95 \\
\hline
\end{tabular}

Table 3: Comparison of gas balances for carbon PFC, tungsten main chamber, all tungsten PFCs.

Fig. 1 : Gas Balance of the shot \#22974 showing the time traces of the different ingredients of Eq.2 (a), the fluxes used for gas balances: the gasinput is defined as sum of the puffed gas and injection by the NBI system, the gas removed is the sum of the fluxes to the cryo, turbo and NBI pumps (b) and the retained inventory (red) and the integral (blue) (c).

Fig. 2: Puffed gas versus the pumped gas of the high gas flux data base shots. The line shows a linear fit to the data. The dashed line is the $100 \%$ line.

Fig. 3: $R_{D}$ (Eq.2) for all discharges of the 2008 campaign showing the initial conditioning.

Fig. 4: Selected discharges show saturation of $R_{D}$ for high gas input. Former shots are inserted for comparison. 

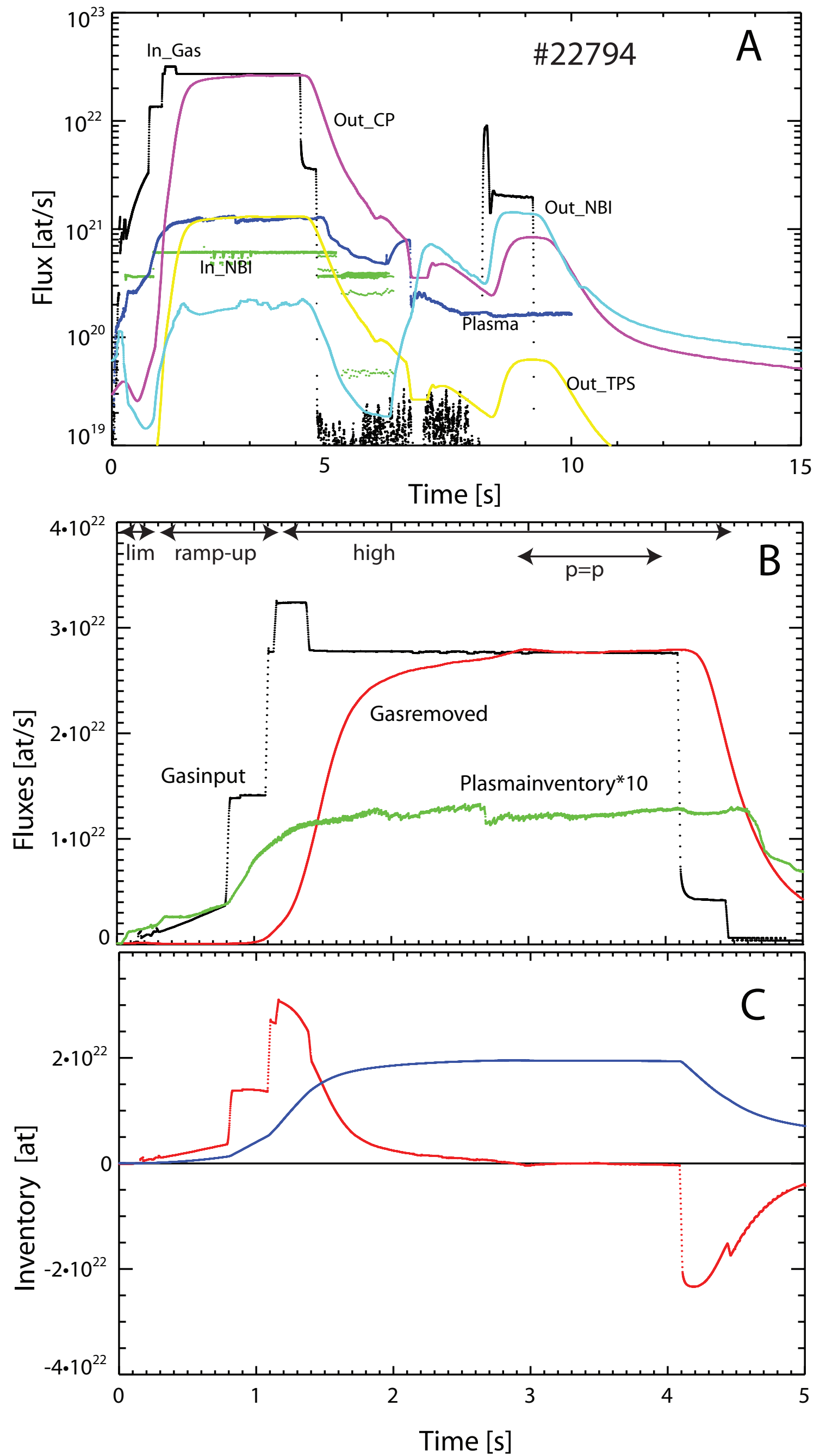

Fig 1 


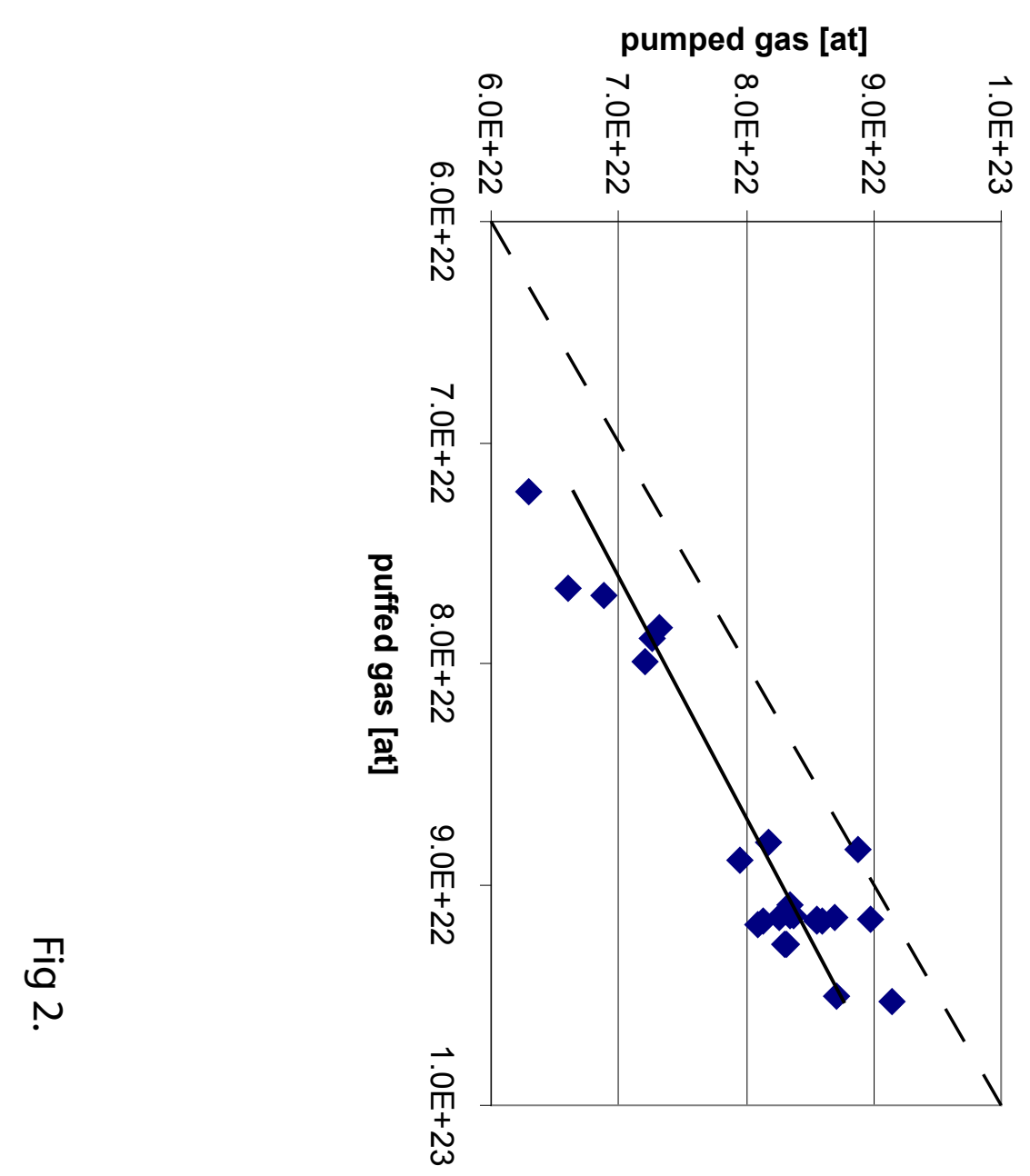




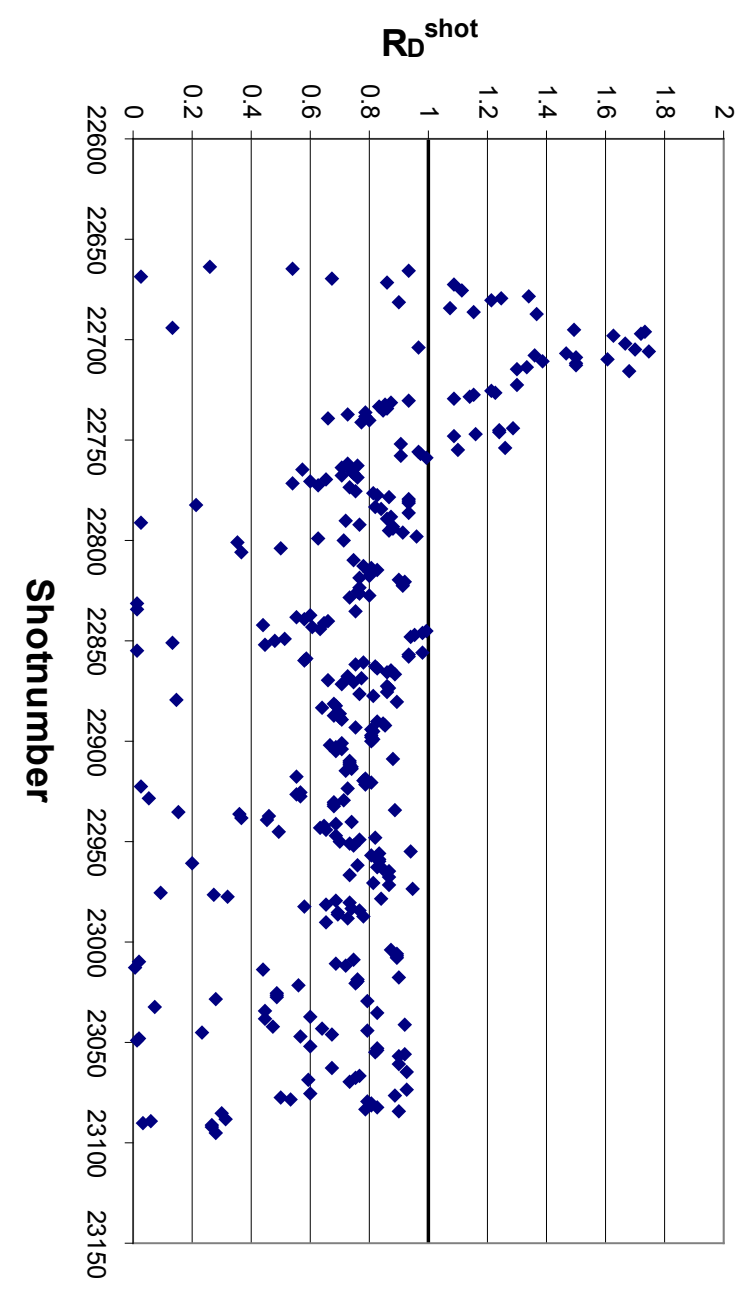




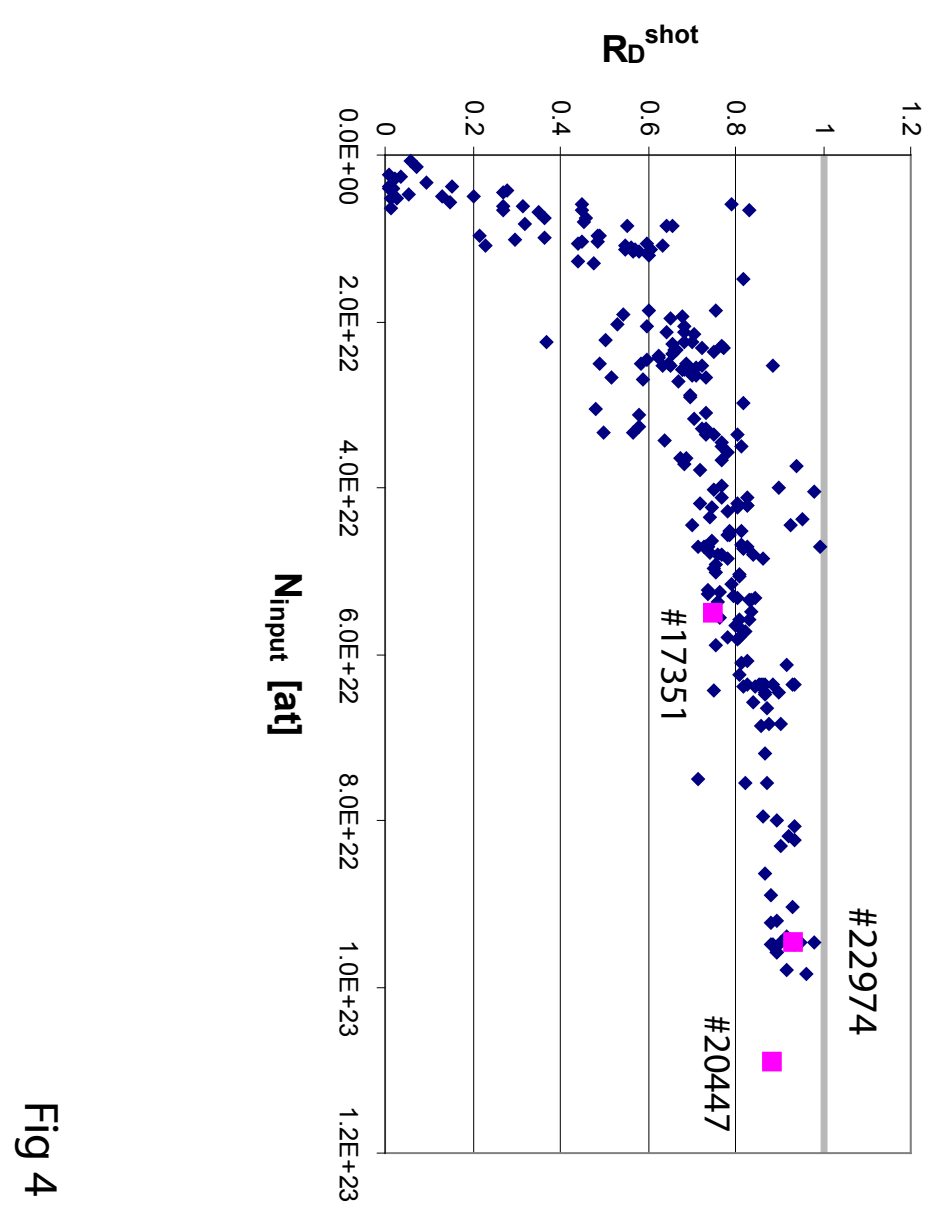

